\title{
Left and Right Inverse Eigenpairs Problem of Orthogonal Matrices
}

\author{
Fanliang Li \\ Institute of Mathematics and Physics, School of Sciences, Central South University of Forestry and Technology, \\ Changsha, China \\ Email:1f1302@tom.com
}

Received September 18, 2012; revised October 18, 2012; accepted October 26, 2012

\begin{abstract}
In this paper, the left and right inverse eigenpairs problem of orthogonal matrices and its optimal approximation solution are considered. Based on the special properties of eigenvalue and the special relations of left and right eigenpairs for orthogonal matrices, we find the equivalent problem, and derive the necessary and sufficient conditions for the solvability of the problem and its general solutions. With the properties of continuous function in bounded closed set, the optimal approximate solution is obtained. In addition, an algorithm to obtain the optimal approximation and numerical example are provided.
\end{abstract}

Keywords: Left and Right Eigenpairs; Orthogonal Matrices; Optimal Approximation

\section{Introduction}

In this paper, we use the following notation. Let $C^{n \times m}$ denote the set of all $n \times m$ complex matrices, $R^{n \times m}$ denote the set of $n \times m$ real matrices, and $R^{n}=R^{n \times 1}$. $A^{\mathrm{T}}, r(A), N(A), R(A), \operatorname{tr}(A)$ and $A^{+}$be the transpose, rank, kernel space, value space, trace and the MoorePenrose generalized inverse of a matrix $A$, respectively. $I_{n}$ is the identity matrix of size $n . O R^{n \times n}$ denotes the set of all $n \times n$ orthogonal matrices, i.e. $A \in O R^{n \times n}$ satisfies $A^{\mathrm{T}} A=A A^{\mathrm{T}}=I_{n}$.

For $A, B \in R^{n \times m},\langle A, B\rangle=\operatorname{tr}\left(B^{\mathrm{T}} \quad A\right)$ denotes the inner product of matrices $A$ and $B$. The induced matrix norm is called Frobenius norm, i.e.

$$
\|A\|=\langle A, A\rangle^{\frac{1}{2}}=\left(\operatorname{tr}\left(\begin{array}{ll}
A^{\mathrm{T}} & A
\end{array}\right)\right)^{\frac{1}{2}},
$$

then $R^{n \times m}$ is a Hilbert inner product space.

The left and right inverse eigenpairs problem is a special inverse eigenvalue problem. That is, for given partial left and right eigenpairs (eigenvalue and corresponding eigenvector)

$$
\left(\gamma_{j}, y_{j}\right), j=1, \cdots, l ;\left(\lambda_{i}, x_{i}\right), i=1, \cdots, h
$$

of matrix $A$, and a special matrix set $S \subseteq C^{n \times n}$, while $h \leq n, l \leq n$ find $A \in S$ such that

$$
\left\{\begin{array}{l}
A x_{i}=\lambda_{i} x_{i}, i=1, \cdots, h, \\
y_{j}^{\mathrm{T}}=\gamma_{j} y_{j}^{\mathrm{T}}, j=1, \cdots, l .
\end{array}\right.
$$

This problem, which usually arise in perturbation analysis of matrix eigenvalue and in recursive matters, have profound application background [1-3]. For different matrix sets $S$, it leads to different left and right inverse eigenpairs problems, such as, Zhang's [4], Li's [5-7], Liang's [8] have considered, respectively, the left and right inverse eigenpairs problem of real matrices, skew-centrosymmetric matrices, generalized centrosymmetric matrices, symmetrizable matrices and generalized reflexive and anti-reflexive matrices, and the explicit expressions of the solutions have been obtained.

Orthogonal matrices have profound applications, such as in matrix singular value decomposition, in matrix norm, in perturbation analysis of matrix eigenvalue, and so on. However, the left and right inverse eigenpairs problem of orthogonal matrices have not been concerned with. In this paper, we will discuss this problem. The orthogonal matrix set $S$ is a bounded closed set, while those matrix sets in [4-8] are subspace. The left and right inverse eigenpairs problems and it's optimal approximation problems for bounded closed set are a new class of left and right inverse eigenpairs problems.

In this paper, we suppose that

$$
\left(\gamma_{j}, y_{j}\right), j=1, \cdots, l ;\left(\lambda_{i}, x_{i}\right), i=1, \cdots, h,
$$

be the left and right eigenpairs of $A$, respectively. If let

$$
\begin{aligned}
& X=\left(x_{1}, \cdots, x_{h}\right) \in C^{n \times h}, \Lambda=\operatorname{diag}\left(\lambda_{1}, \cdots, \lambda_{h}\right) \in C^{h \times h} \\
& Y=\left(y_{1}, \cdots, y_{l}\right) \in C^{n \times l}, \Gamma=\operatorname{diag}\left(\gamma_{1}, \cdots, \gamma_{l}\right) \in C^{l \times l}
\end{aligned}
$$


then the problems studied in this paper can be described as follows.

\section{Problem I Giving}

$$
\begin{aligned}
& X \in C^{n \times h}, \Lambda=\operatorname{diag}\left(\lambda_{1}, \cdots, \lambda_{h}\right) \in C^{h \times h} \\
& Y \in C^{n \times l}, \Gamma=\operatorname{diag}\left(\gamma_{1}, \cdots, \gamma_{l}\right) \in C^{l \times l}
\end{aligned}
$$

find $A \in O R^{n \times n}$ such that

$$
\left\{\begin{array}{c}
A X=X \Lambda, \\
Y^{\mathrm{T}} A=\Gamma Y^{\mathrm{T}} .
\end{array}\right.
$$

Problem II Giving $A^{*} \in R^{n \times n}$, finding $\hat{A} \in S_{E}$ such that

$$
\left\|A^{*}-\hat{A}\right\|=\min _{\forall A \in S_{E}}\left\|A^{*}-A\right\|,
$$

where $S_{E}$ is the solution set of Problem I.

This paper is organized as follows. In Section 2, we first study the special properties of eigenvalue of orthogonal matrices. Then with these properties, we find the equivalent problem of Problem I and obtain the solvability conditions and the general solutions of Problem I. In Section 3, we first prove that the approximation solution of Problem II exist and can be obtained by applying the properties of continuous function in bounded closed set. Then we obtain the approximation solution of Problem II. Finally, the algorithm and example to obtain the approximation solution are given.

\section{Solvability Conditions of Problem I}

First, we discuss the properties of orthogonal matrices.

Lemma 1 [9] If $A \in R^{n \times n}$, then there is a matrix $Q \in O R^{n \times n}$, and a block upper triangular matrix $R \in R^{n \times n}$ such that

$$
Q^{\mathrm{T}} A Q=R,
$$

where each diagonal block of $R$ is $1 \times 1$ block or $2 \times 2$ block, and every $1 \times 1$ block correspond a real eigenvalue of $A$, every $2 \times 2$ block correspond a pair of conjugate imaginary eigenvalue of $A$.

From the definition of orthogonal matrices and Lemma 1 , it is easy to obtain the following lemma.

Lemma 2 If $A \in O R^{n \times n}$, then there is a matrix $Q \in O R^{n \times n}$, and a block diagonal matrix $R \in R^{n \times n}$ such that

$$
Q^{\mathrm{T}} A Q=R,
$$

where each diagonal block of $R$ is $1 \times 1$ block or $2 \times 2$ block, and every $1 \times 1$ block is $(1)$ or $(-1)$, every $2 \times 2$ block can be denoted as follows.

$$
\left(\begin{array}{cc}
\cos \theta & \sin \theta \\
-\sin \theta & \cos \theta
\end{array}\right), \theta \neq k 180^{\circ}, k \in Z .
$$

From Lemma 2, it is easy to obtain the following conclusions.
1) $R \in O R^{n \times n}$.

2) If $A \in O R^{n \times n}$, then the module of eigenvalue of $A$ is 1. Namely, the eigenvalues of $A$ distribute on the unit circle.

3) If $A \in O R^{n \times n}$, then the imaginary eigenvalue of $A$ can be denoted as follows.

$$
\cos \theta+\mathrm{i} \sin \theta, \theta \neq k 180^{\circ}, k \in Z,
$$

where $\mathrm{i}$ denote the imaginary unit, i.e. $\mathrm{i}^{2}=-1$. If $\lambda=\cos \theta+i \sin \theta$ is an imaginary eigenvalue of $A$, $u+\mathrm{i} v$ is a corresponding eigenvector of $\lambda$, where $u \in R^{n}, v \in R^{n}$. It is clear that $\bar{\lambda}=\cos \theta-i \sin \theta$ is also an imaginary eigenvalue of $A$, and $u-i v$ is a corresponding eigenvector of $\bar{\lambda}$. This gives

$$
A\left(\begin{array}{ll}
u & v
\end{array}\right)=\left(\begin{array}{ll}
u & v
\end{array}\right)\left(\begin{array}{cc}
\cos \theta & \sin \theta \\
-\sin \theta & \cos \theta
\end{array}\right) .
$$

Lemma 3 Let $A \in O R^{n \times n}$, if $(\lambda, x)$ is a right eigenpairs of $A$, then $\left(\lambda^{-1}, x\right)$ is a left eigenpairs of $A$.

Proof If $(\lambda, x)$ is a right eigenpairs of $A$, then we have

$$
A x=\lambda x, A^{\mathrm{T}} A x=\lambda A^{\mathrm{T}} x,
$$

Combining $A^{\mathrm{T}} A=A A^{\mathrm{T}}=I_{n}$, we have $A^{\mathrm{T}} x=\lambda^{-1} x$.

Therefore, $\left(\lambda^{-1}, x\right)$ is a left eigenpairs of $A$.

According to Lemma 2 and its conclusions, in Lemma 3 , if $\lambda= \pm 1$, then $\lambda^{-1}= \pm 1$; if $\lambda=\cos \theta \pm i \sin \theta$, and the eigenvector corresponding to $\lambda$ is $u \pm \mathrm{i} v$, then

$$
\lambda^{-1}=\cos \theta \mp i \sin \theta \text {. }
$$

Combining $A^{\mathrm{T}} x=\lambda^{-1} x$, we have

$$
A^{\mathrm{T}}\left(\begin{array}{ll}
u & v
\end{array}\right)=\left(\begin{array}{ll}
u & v
\end{array}\right)\left(\begin{array}{cc}
\cos \theta & -\sin \theta \\
\sin \theta & \cos \theta
\end{array}\right)
$$

According to the analysis before, in Problem I, we can suppose as follows.

$$
\begin{aligned}
& X=\left(X_{1}, \cdots, X_{t}, X_{t_{1}}, X_{h-2 t-t_{1}}\right), X_{j} \in R^{n \times 2}, \\
& \Lambda=\operatorname{diag}\left(B_{1}, \cdots, B_{t}, I_{t_{1}},-I_{h-2 t-t_{1}}\right) \\
& B_{j}=\left(\begin{array}{cc}
\cos \theta_{j} & \sin \theta_{j} \\
-\sin \theta_{j} & \cos \theta_{j}
\end{array}\right) \in O R^{2 \times 2}, \\
& \theta_{j} \neq k 180^{\circ}, k \in Z, j=1, \cdots, t \\
& Y=X, \Gamma=\Lambda^{\mathrm{T}}, \Lambda \in O R^{h \times h} .
\end{aligned}
$$

Let the svd of $X$ in Problem I as follows.

$$
\begin{aligned}
& X=U\left(\begin{array}{ll}
\Sigma & 0 \\
0 & 0
\end{array}\right) V^{\mathrm{T}}=\left(U_{1}, U_{2}\right)\left(\begin{array}{cc}
\Sigma & 0 \\
0 & 0
\end{array}\right)\left(\begin{array}{c}
V_{1}^{\mathrm{T}} \\
V_{2}^{\mathrm{T}}
\end{array}\right)=U_{1} \Sigma V_{1}^{\mathrm{T}} \\
& U \in O R^{n \times n}, V \in O R^{h \times h}, U_{1} \in R^{n \times r}, V_{1} \in R^{h \times r} \\
& \Sigma=\operatorname{diag}\left(\alpha_{1}, \cdots, \alpha_{r}\right), \\
& \alpha_{i}>0, i=1, \cdots, r, r=r(X) .
\end{aligned}
$$


Denote

$$
\begin{aligned}
& U^{\mathrm{T}} A U=\left(\begin{array}{ll}
A_{11} & A_{12} \\
A_{21} & A_{22}
\end{array}\right), A_{i j}=U_{i}^{\mathrm{T}} A U_{j}, i, j=1,2 . \\
& V^{\mathrm{T}} \Lambda V=\left(\begin{array}{ll}
\Lambda_{11} & \Lambda_{12} \\
\Lambda_{21} & \Lambda_{22}
\end{array}\right), \Lambda_{i j}=V_{i}^{\mathrm{T}} \Lambda V_{j}, i, j=1,2 .
\end{aligned}
$$

Theorem 1 If $X, Y, \Lambda, \Gamma$ are given by (2.1) and the svd of $X$ is given by (2.2), then Problem $I$ has a solution $A \in O R^{n \times n}$ if and only if

$$
\begin{aligned}
\Lambda X^{\mathrm{T}} X & =X^{\mathrm{T}} X \Lambda, X \Lambda=X \Lambda X^{+} X, X \Lambda X^{+} \\
& =\left(X \Lambda X^{+}\right)^{\mathrm{T}}
\end{aligned}
$$

Moreover, the general solution can be expressed as

$$
\begin{aligned}
& A=X \Lambda X^{+}+U_{2} P U_{2}{ }^{\mathrm{T}}, \forall P \in O R^{(n-r) \times(n-r)}, \\
& R\left(U_{2}\right)=N\left(X^{\mathrm{T}}\right)
\end{aligned}
$$

Proof (2.1) implies that Problem I has a solution $A \in O R^{n \times n}$ if and only if matrix equations $A X=X \Lambda$, $A^{\mathrm{T}} X=X \Lambda$ has a solution $A \in O R^{n \times n}$.

Necessity: If matrix equations $A X=X \Lambda, A^{\mathrm{T}} X=X \Lambda$ has a solution $A \in O R^{n \times n}$, then it is easy to obtain that $X^{\mathrm{T}} A^{\mathrm{T}} A X=\Lambda^{\mathrm{T}} X^{\mathrm{T}} X \Lambda$. This implies that

$$
\Lambda X^{\mathrm{T}} X=X^{\mathrm{T}} X \Lambda
$$

Combining (2.2) and $A X=X \Lambda, A^{\mathrm{T}} X=X \Lambda$, we have

$$
\left\{\begin{array}{l}
A U\left(\begin{array}{ll}
\Sigma & 0 \\
0 & 0
\end{array}\right) V^{\mathrm{T}}=U\left(\begin{array}{ll}
\Sigma & 0 \\
0 & 0
\end{array}\right) V^{\mathrm{T}} \Lambda, \\
A^{\mathrm{T}} U\left(\begin{array}{ll}
\Sigma & 0 \\
0 & 0
\end{array}\right) V^{\mathrm{T}}=U\left(\begin{array}{ll}
\Sigma & 0 \\
0 & 0
\end{array}\right) V^{\mathrm{T}} \Lambda .
\end{array}\right.
$$

i.e.

$$
\left\{\begin{array}{c}
U A U\left(\begin{array}{ll}
\Sigma & 0 \\
0 & 0
\end{array}\right)=\left(\begin{array}{ll}
\Sigma & 0 \\
0 & 0
\end{array}\right) V^{\mathrm{T}} \Lambda V, \\
U^{\mathrm{T}} A^{\mathrm{T}} U\left(\begin{array}{ll}
\Sigma & 0 \\
0 & 0
\end{array}\right)=\left(\begin{array}{ll}
\Sigma & 0 \\
0 & 0
\end{array}\right) V^{\mathrm{T}} \Lambda V .
\end{array}\right.
$$

According to (2.3) and (2.4), we have

$$
\left\{\begin{array}{l}
\left(\begin{array}{ll}
A_{11} & A_{12} \\
A_{21} & A_{22}
\end{array}\right)\left(\begin{array}{ll}
\Sigma & 0 \\
0 & 0
\end{array}\right)=\left(\begin{array}{ll}
\Sigma & 0 \\
0 & 0
\end{array}\right)\left(\begin{array}{ll}
\Lambda_{11} & \Lambda_{12} \\
\Lambda_{21} & \Lambda_{22}
\end{array}\right), \\
\left(\begin{array}{ll}
A_{11}{ }^{\mathrm{T}} & A_{12}{ }^{\mathrm{T}} \\
A_{21}{ }^{\mathrm{T}} & A_{22}{ }^{\mathrm{T}}
\end{array}\right)\left(\begin{array}{ll}
\Sigma & 0 \\
0 & 0
\end{array}\right)=\left(\begin{array}{ll}
\Sigma & 0 \\
0 & 0
\end{array}\right)\left(\begin{array}{ll}
\Lambda_{11} & \Lambda_{12} \\
\Lambda_{21} & \Lambda_{22}
\end{array}\right) .
\end{array}\right.
$$

This gives

$$
\begin{aligned}
& A_{11} \Sigma=\Sigma \Lambda_{11}, \Sigma \Lambda_{12}=0, A_{21} \Sigma=0, \\
& A_{11}{ }^{\mathrm{T}} \Sigma=\Sigma \Lambda_{11}, A_{12}{ }^{\mathrm{T}} \Sigma=0 .
\end{aligned}
$$

i.e.

$$
\begin{gathered}
A_{11}=\Sigma V_{1}^{\mathrm{T}} \Lambda V_{1} \Sigma^{-1}, \Sigma V_{1}^{\mathrm{T}} \Lambda V_{1} \Sigma^{-1}=\Sigma^{-1} V_{1}^{\mathrm{T}} \Lambda^{\mathrm{T}} V_{1} \Sigma, \\
V_{1} \Lambda V_{2}^{\mathrm{T}}=0, A_{21}=0, A_{12}=0 . \\
V_{1} \Lambda V_{2}^{\mathrm{T}}=0 \text { gives } \\
X \Lambda=X \Lambda X^{+} X . \\
\Sigma V_{1}^{\mathrm{T}} \Lambda V_{1} \Sigma^{-1}=\Sigma^{-1} V_{1}^{\mathrm{T}} \Lambda^{\mathrm{T}} V_{1} \Sigma \text { gives } \\
X \Lambda X^{+}=\left(X \Lambda^{\mathrm{T}} X^{+}\right)^{\mathrm{T}} .
\end{gathered}
$$

Combining (2.7), (2.8) and (2.9), we obtain (2.5).

Sufficiency: Give $P_{0} \in O R^{(n-r) \times(n-r)}$ and let

$$
A_{0}=U\left(\begin{array}{cc}
\Sigma V_{1}^{\mathrm{T}} \Lambda V_{1} \Sigma^{-1} & 0 \\
0 & P_{0}
\end{array}\right) U^{\mathrm{T}} .
$$

If let $A_{11}=\Sigma V_{1}^{\mathrm{T}} \Lambda V_{1} \Sigma^{-1}$, then from $\Lambda X^{\mathrm{T}} X=X^{\mathrm{T}} X \Lambda$, it is easy to obtain

$$
A_{11}{ }^{\mathrm{T}} A_{11}=A_{11} A_{11}{ }^{\mathrm{T}}=I_{r} .
$$

Combining (2.10) and (2.11), it is easy to obtain $A_{0}{ }^{\mathrm{T}} A_{0}=A_{0} A_{0}^{\mathrm{T}}=I_{n}$, i.e. $A_{0} \in O R^{n \times n}$. Combining (2.2), (2.8) and (2.10), it is easy to obtain $A_{0} X=X \Lambda$. Combining (2.2), (2.9) and (2.10), it is easy to obtain $A_{0}{ }^{\mathrm{T}} X=X \Lambda^{\mathrm{T}}$. So, $A_{0}$ is a solution of Problem I. It is clear that for any $P \in O R^{(n-r) \times(n-r)}$,

$$
\begin{aligned}
A & =U\left(\begin{array}{cc}
\Sigma V_{1}^{\mathrm{T}} \Lambda V_{1} \Sigma^{-1} & 0 \\
0 & P
\end{array}\right) U^{\mathrm{T}} \\
& =X \Lambda X^{+}+U_{2} P U_{2}^{\mathrm{T}}
\end{aligned}
$$

is the general solution of Problem $I$.

\section{The Solution of Problem II}

According to (2.6), it is easy to prove that if Problem I has a solution $A \in O R^{n \times n}$, then the solution set $S_{E}$ is a nonempty bounded closed set, and Frobinus norm is the continuous function of matrix. According to the properties of continuous function in bounded closed set (There exist the minimal value and the maximal value for continuous function in bounded closed set), we can claim that for any given $A^{*} \in R^{n \times n}$, there exists the optimal approximation for Problem II. Moreover, we can obtain the optimal approximation solution of Problem II.

Lemma 4 If giving $X \in R^{n \times h}, n \geq h$, let the svd of $X$ is given by (2.2), $O R^{n \times h}$ denotes the column orthogonal matrix set (It is easy to see that if $n=h$, then $O R^{n \times n}$ is a subset of $\left.O R^{n \times n}\right)$. Then the solution set of the following problem

is

$$
\min _{\forall K \in O R^{n \times h}}\|X-K\|
$$

$$
E(X)=\left\{U\left(\begin{array}{cc}
I_{r} & 0 \\
0 & M
\end{array}\right) V^{\mathrm{T}} \mid M \in O R^{(n-r) \times(h-r)}\right\} .
$$


Proof Let $U^{\mathrm{T}} K V=\left(\begin{array}{ll}K_{11} & K_{12} \\ K_{21} & K_{22}\end{array}\right)$, combining (2.2), we have

$$
\begin{aligned}
\|X-K\|^{2} & =\left\|U\left(\begin{array}{ll}
\Sigma & 0 \\
0 & 0
\end{array}\right) V^{\mathrm{T}}-K\right\|^{2} \\
& =\left\|\left(\begin{array}{ll}
\Sigma & 0 \\
0 & 0
\end{array}\right)-\left(\begin{array}{ll}
K_{11} & K_{12} \\
K_{21} & K_{22}
\end{array}\right)\right\|^{2} \\
& =\left\|\Sigma-K_{11}\right\|^{2}+\left\|K_{12}\right\|^{2}+\left\|K_{21}\right\|^{2}+\left\|K_{22}\right\|^{2} .
\end{aligned}
$$

This implies $\|X-K\|=\min$ if and only if $\left\|\Sigma-K_{11}\right\|=\min ,\left\|K_{12}\right\|=\min ,\left\|K_{21}\right\|=\min ,\left\|K_{22}\right\|=\min$, combining $K \in O R^{n \times h}$, we have

$$
K_{11}=I_{r}, K_{12}=0, K_{21}=0, K_{22} \in O R^{(n-r) \times(h-r)} .
$$

This gives the conclusion.

Theorem 2 Giving $A^{*} \in R^{n \times n}$, if $X, \Lambda, Y, \Gamma$ are given by (2.1), and satisfy the conditions of Theorem 1 , then there exist solutions for Problem II. Moreover, solutions can be expressed as follows.

$$
A=X \Lambda X^{+}+U_{2} P U_{2}{ }^{\mathrm{T}}, P \in E\left(U_{2} A^{*} U_{2}{ }^{\mathrm{T}}\right) .
$$

Proof According to Theorem 1, $\forall A \in S_{E}$ we have

$$
\begin{aligned}
\left\|A^{*}-A\right\|^{2} & =\left\|A^{*}-X \Lambda X^{+}-U_{2} P U_{2}^{\mathrm{T}}\right\|^{2} \\
& =\left\|A^{*}-U\left(\begin{array}{cc}
\Sigma V_{1}^{\mathrm{T}} \Lambda V_{1} \Sigma^{-1} & 0 \\
0 & P
\end{array}\right) U^{\mathrm{T}}\right\|^{2} \\
& =\left\|U^{\mathrm{T}} A^{*} U-\left(\begin{array}{cc}
\Sigma V_{1}^{\mathrm{T}} \Lambda V_{1} \Sigma^{-1} & 0 \\
0 & P
\end{array}\right)\right\|^{2} .
\end{aligned}
$$

Let

$$
U^{\mathrm{T}} A^{*} U=\left(\begin{array}{ll}
A_{11}^{*} & A_{12}^{*} \\
A_{21}^{*} & A_{22}^{*}
\end{array}\right), A_{i j}^{*}=U_{i}^{\mathrm{T}} A^{*} U_{j}, i, j=1,2 .
$$

Hence, we have

$$
\begin{aligned}
\left\|A^{*}-A\right\|^{2} & =\left\|\left(\begin{array}{cc}
A_{11}^{*}-\Sigma V_{1}^{\mathrm{T}} \Lambda V_{1} \Sigma^{-1} & A_{12}^{*} \\
A_{21}^{*} & A_{22}^{*}
\end{array}\right)\right\|^{2} \\
& =\left\|A_{11}^{*}-\Sigma V_{1}^{\mathrm{T}} \Lambda V_{1} \Sigma^{-1}\right\|^{2}+\left\|A_{12}^{*}\right\|^{2} \\
& +\left\|A_{21}^{*}\right\|^{2}+\left\|A_{22}^{*}-P\right\|^{2} .
\end{aligned}
$$

This implies that

if and only if

$$
\min _{\forall A \in S_{E}}\left\|A^{*}-A\right\|
$$

$$
\min _{\forall P \in O R^{(n-r) \times(n-r)}}\left\|A_{22}^{*}-P\right\| .
$$

From Lemma 4, it is easy to prove that the solution of problem

$$
\min _{\forall P \in O R^{(n-r) \times(n-r)}}\left\|A_{22}^{*}-P\right\|
$$

is $P \in E\left(A_{22}^{*}=U_{2} A^{*} U_{2}^{\mathrm{T}}\right)$. This gives the conclusion.

\section{Algorithm}

1) Give $A^{*}$, and according to (2.1), input $X, \Lambda, Y, \Gamma$.

2) Compute the svd of $X$.

3) Compute $\Lambda X^{\mathrm{T}} X, X^{\mathrm{T}} X \Lambda, X \Lambda, X \Lambda X^{+} X, \quad X \Lambda^{\mathrm{T}}$, $X \Lambda^{\mathrm{T}} X^{+} X, X \Lambda X^{+}, X \Lambda^{\mathrm{T}} X^{+}$, if (2.5) holds, then go to 4; otherwise stop.

4) Compute the svd of $U_{2}{ }^{\mathrm{T}} A^{*} U_{2}$.

5) Give a matrix $M$ which satisfies $M^{\mathrm{T}} M=I_{n-r}$, and compute $E\left(U_{2}{ }^{\mathrm{T}} A^{*} U_{2}\right)$ according to (3.1).

6) According to (3.2) calculate $\hat{A}$.

\section{Example $(n=8, h=l=5)$}

According to (2.1), input $X, \Lambda, Y, \Gamma$ as follows.

$X=Y=$

$\left(\begin{array}{ccccc}0.6269 & 0 & -0.0517 & -0.1481 & 0 \\ 0.0826 & 0.4354 & -0.3680 & 0.1739 & 0 \\ 0.0747 & 0.2439 & 0.5914 & 0 & 0 \\ 0.1657 & 0.1401 & 0.0376 & -0.0941 & 0.4412 \\ -0.0256 & -0.0107 & -0.0713 & -0.5529 & -0.4828 \\ 0.1659 & -0.3668 & 0.0120 & 0.2793 & -0.3220 \\ 0.1833 & 0.0315 & 0.0626 & 0.2180 & -0.3970 \\ -0.0730 & 0.3094 & 0.0399 & 0.0877 & -0.5577\end{array}\right)$
$\Lambda=\Gamma^{\mathrm{T}}=\left(\begin{array}{ccccc}-0.7115 & -0.7027 & 0 & 0 & 0 \\ 0.7027 & -0.7115 & 0 & 0 & 0 \\ 0 & 0 & 0.2670 & -0.9637 & 0 \\ 0 & 0 & 0.9637 & 0.2670 & 0 \\ 0 & 0 & 0 & 0 & 1\end{array}\right)$

$A^{*}=$

$$
\left(\begin{array}{cccccccc}
4.7 & -3.5 & 3.9 & -2.3 & 2.6 & -1.9 & -3.6 & 5.1 \\
-2.9 & 2.8 & 0.8 & 2.8 & 1.7 & 4.5 & 7.3 & 6.9 \\
1.3 & 1.6 & 1.2 & 1.6 & 2.5 & 2.1 & 9.5 & -2.9 \\
9.6 & -2.1 & -1.5 & -2.5 & 2.8 & 0.7 & 2.1 & 7.5 \\
-1.5 & 1.4 & 7.5 & -0.1 & 0.5 & -3.5 & -1.5 & 1.6 \\
2.7 & -1.8 & 9.6 & 0.2 & 1.9 & 6.9 & 3.8 & -3.1 \\
1.7 & 3.2 & -1.7 & -2.1 & 2.3 & 1.4 & 1.9 & 7.6 \\
8.1 & 5.5 & 2.2 & 7.3 & 4.3 & 2 & 2.6 & 1.5
\end{array}\right)
$$

Compute the svd of $X$ and Compute $\Lambda X^{\mathrm{T}} X$, $X^{\mathrm{T}} X \Lambda, X \Lambda, X \Lambda X^{+} X, X \Lambda^{\mathrm{T}}, X \Lambda^{\mathrm{T}} X^{+} X, X \Lambda X^{+}, X \Lambda^{\mathrm{T}} X^{+}$, it is clear that (2.5) holds. Using the software "MATLAB", we can obtain the solution $\hat{A}$ of Problem II as follows. 


$$
\left(\begin{array}{cccccccc}
-0.4918 & -0.1887 & -0.3249 & -0.5601 & 0.0580 & 0.2089 & -0.3528 & -0.3628 \\
0.0076 & -0.1584 & -0.0800 & 0.1126 & -0.3338 & 0.5430 & 0.6329 & -0.3860 \\
0.1653 & -0.4241 & 0.1155 & 0.1948 & 0.7162 & -0.1305 & 0.0860 & -0.4518 \\
-0.0256 & -0.3581 & -0.3853 & 0.6559 & -0.1731 & 0.2005 & -0.4625 & 0.0911 \\
-0.0043 & 0.3183 & -0.7367 & 0.0260 & 0.4331 & 0.0564 & 0.3168 & 0.2534 \\
-0.7586 & -0.0182 & 0.3543 & 0.2512 & 0.2566 & 0.1943 & 0.1691 & 0.3214 \\
0.0430 & -0.7270 & -0.1162 & -0.3012 & -0.1036 & -0.2061 & 0.2753 & 0.4863 \\
0.3907 & -0.0308 & 0.2110 & -0.2254 & 0.2793 & 0.7217 & -0.2217 & 0.3210
\end{array}\right) .
$$

\section{Conclusions}

The left and right inverse eigenpairs problem is a special inverse eigenvalue problem. Different matrix set can lead to different left and right inverse eigenpairs problems, such as, Zhang's [4], Li's [5-7], Liang's [8] have considered, respectively, the left and right inverse eigenpairs problem of real matrices, skew-centrosymmetric matrices, generalized centrosymmetric matrices, symmetrizable matrices and generalized reflexive and anti-reflexive matrices, and the explicit expressions of the solutions have been obtained. In this paper, we considered the left and right inverse eigenpairs problem of orthogonal matrices (namely Problem I) and its optimal approximation problem (namely Problem II). Based on the special properties of eigenvalue and the special relations of left and right eigenpairs for orthogonal matrices, we find the equivalent problem, and derive the necessary and sufficient conditions for the solvability of the problem I and its general solutions. The orthogonal matrix set is a bounded closed set. We obtain the optimal approximate solution with the properties of continuous function in bounded closed set. Compare the problems in [4-8] (those matrix sets are subspace), the bounded closed set problems we discussed in this paper are a new class of left and right inverse eigenpairs problems.

\section{Acknowledgements}

The authors are very grateful to thank the referee for their valuable comments. They also thank editors for their helpful suggestions.

This research was supported by National natural Science Foundation of China (31170532).

\section{REFERENCES}

[1] J. H. Wilkinson, "The Algebraic Eigenvalue Problem," Oxford University Press, Oxford, 1965.

[2] M. Arav, D. Hershkowitz, V. Mehrmann, et al., "The Recursive Inverse Eigenvalue Problem," SIAM Journal on Matrix Analysis and Applications, Vol. 22, No. 2, 2000, pp. 392-412.

[3] R. Loewy and V. Mehrmann, "A Note on the Symmetric Recursive Inverse Eigenvalue Problem," SIAM Journal on Matrix Analysis and Applications, Vol. 25, No. 1, 2003, pp. 180-187.

[4] L. Zhang and D. X. Xie, "A Class of Inverse Eigenvalue Problems," Acta Mathematica Scientia, Vol. 1, No. 13, 1993, pp. 94-99.

[5] F. L. Li, X. Y. Hu and L. Zhang, "Left and Right Eigenpairs Problem of Skew-Centrosymmetric Matrices," Applied Mathematics and Computation, Vol. 177, No. 1, 2006, pp. 105-110.

[6] F. L. Li, X. Y. Hu and L. Zhang, "Left and Right Inverse Eigenpairs Problem of Generalized Centrosymmetric Matrices and Its Optimal Approximation Problem," Applied Mathematics and Computation, Vol. 212, No. 1, 2009, pp. 481-487. doi:10.1016/j.amc.2009.02.035

[7] F. L. Li and K. K. Zhang, "Left and Right Inverse Eigenpairs Problem for the Symmetrizable Matrices," Proceedings of the Ninth International Conference on Matrix Theory and Its Applications, Vol. 1, 2010, pp. 179-182.

[8] M. L. Liang and L. F. Dai, "The Left and Right Inverse Eigenvalue Problems of Generalized Reflexive and AntiReflexive Matrices," Journal of Computational and Applied Mathematics, Vol. 234, No. 3, 2010, pp. 743-749.

[9] H. Dai, "The Theory of Matrices," Science Press, Beijing, 2001. 